\title{
200. Sitzung der Zentralen Kommission für die Biologische Sicherheit
}

\author{
Wilfried Wackernagel ${ }^{1} \cdot$ Inge Kruczek $^{2}$
}

(C) Bundesamt für Verbraucherschutz und Lebensmittelsicherheit (BVL) 2016

Seit Inkrafttreten des Gentechnikgesetzes (GenTG) am 1. Juli 1990, tagt die Zentrale Kommission für die Biologische Sicherheit (ZKBS) am 7. Juni 2016 zum 200. Mal. Das Treffen findet im Bundesamt für Verbraucherschutz und Lebensmittelsicherheit (BVL) in Berlin statt. Die ZKBS gibt es aber schon wesentlich länger, nämlich seit dem 15. Februar 1978, als die erste Fassung der ,,Richtlinien zum Schutz vor Gefahren durch in vitro neukombinierte Nukleinsäuren" in Kraft trat. Wilfried Wackernagel (WW) und Inge Kruczek (IK) erinnern sich.

IK: Ich kam im September 1990 zur Geschäftsstelle der ZKBS nach Berlin. Die erste Sitzung der ZKBS, an der ich teilnahm, fand am 6. November 1990 statt. Es war aber bereits die zweite Sitzung der ZKBS nach Inkrafttreten des GenTG. In dieser Sitzung hat die ZKBS neun Anträge auf Sicherheitseinstufung gentechnischer Arbeiten aus sehr verschiedenen fachlichen Richtungen beraten, außerdem noch einen Antrag auf Genehmigung einer Freisetzung gentechnisch veränderter Petunien. Ich fand die Diskussionen sehr interessant und anregend, weil ich vorher als experimentelle Virologin hauptsächlich im

Inge Kruczek: Bis Januar 2016: Geschäftsstelle der ZKBS, Leiterin der Referates "Sicherheitsempfehlungen und ZKBS" am Bundesamt für Verbraucherschutz und Lebensmittelsicherheit, Berlin, Germany.

Inge Kruczek

kruczek@berlin.de

1 Institut für Biologie und Umweltwissenschaften, Mitglied der ZKBS für den Bereich Gewerkschaft, Universität Oldenburg, Oldenburg, Germany

2

Tulpenstraße 31, 16548 Glienicke, Germany
Labor gearbeitet hatte. Damals war Professor Peter Starlinger von der Universität Köln noch Vorsitzender der ZKBS. Herr Wackernagel, Sie sind im September 1991 als Professor der Genetik an der Universität Oldenburg zum Mitglied in der ZKBS für den Bereich Gewerkschaften berufen worden. Können Sie sich noch an Ihre erste Sitzung erinnern?

WW: Ja, sehr gut sogar. Das war dann schon die 16 . Sitzung der ZKBS, im Dezember 1991. Die Sitzungen fanden fast monatlich statt, manchmal sogar öfter. Wir tagten noch im Arzneimittelinstitut in der Seestraße in einem Raum ohne Außenlicht, wodurch für mein Empfinden eine besondere Konzentration auf die Sachinhalte eintrat. Wir haben auf dieser Sitzung Forschungsarbeiten zu HIV bewertet, dessen Gefährdungspotenzial damals noch umstritten war. Außerdem haben wir sehr ausführlich über den Entwurf einer Geschäftsordnung für die ZKBS diskutiert. Bei dieser Sitzung war dann schon Professor Gerd Hobom von der Universität Gießen Vorsitzender der ZKBS. Das blieb er bis zum Jahr 2000. Dann übernahm Professor Klaus-Peter Schaal von der Universität Bonn den Vorsitz. Die Geschäftsordnung wurde übrigens nach rechtlicher Prüfung in der 19. Sitzung im April 1992 verabschiedet.

IK: Ja, und sie gilt auch heute noch. Von 2007 bis Mai dieses Jahres war dann Professor Herbert Pfister von der Universität Köln Vorsitzender. Und nun wurde erstmalig eine Frau von der ZKBS zur Vorsitzenden gewählt: Professor Sigrun Smola von der Universität des Saarlandes. Unsere Vorsitzenden haben immer bis zum Ende ihrer Amtszeit an der Universität und ihrer Berufungszeit in der ZKBS „durchgehalten“ und 
die Sitzungen auch durch schwierige Diskussionen souverän geleitet.

Welche Diskussionen haben Sie im Verlauf Ihrer langjährigen Mitgliedschaft als besonders interessant empfunden?

WW: Ich habe tatsächlich die meisten Diskussionen als sehr fesselnd und immer als beispielhaft sachlich erlebt. Es sind ja Mitglieder und stellvertretende Mitglieder sehr unterschiedlicher fachlicher Richtungen vertreten, die ihre jeweilige Expertise in die Diskussionen einbringen. Wir haben mittlerweile über 1900 gentechnische Arbeiten bewertet, neun davon wurden der höchsten Sicherheitsstufe, der Sicherheitsstufe 4, zugeordnet. Das waren, unter anderem, gentechnische Arbeiten mit dem Ebolavirus. Aber auch die Zuordnung in die Sicherheitsstufe 1 ist aus fachlicher Sicht eine Herausforderung, denn nur solche gentechnischen Arbeiten, bei denen wirklich kein Risiko für Menschen, Tiere, Pflanzen, Umwelt und Sachgüter vorhanden ist, werden der Sicherheitsstufe 1 zugeordnet. Und das ist zu überprüfen. Bei Arbeiten mit infektiösen Erregern werden Sicherheitsmaßnahmen festgelegt, durch die eine akzidentelle Infektion verhindert werden soll.

Bei der Behandlung von Freisetzungsanträgen gelten dagegen noch andere Ziele, weil hierbei Umweltaspekte im Vordergrund stehen: durch geeignete Maßnahmen soll die zeitliche und räumliche Begrenzung der Freisetzung der genetisch veränderten Organismen sichergestellt werden, seien es Bakterien oder Pflanzen. Die gentechnisch veränderten Pflanzen zum Beispiel sollen unter anderem nicht auskreuzen oder überdauern können. Allerdings haben wir seit drei Jahren keine Freisetzungsanträge mehr vorgelegt bekommen und nur noch wenige Anträge auf Inverkehrbringen. Im Jahr 1999 waren es dagegen allein noch 20 Freisetzungsanträge, so dass Sondersitzungen des vorbereitenden Arbeitskreises notwendig waren.

IK: Auch die Synthetische Biologie ist ein Forschungsfeld, mit dem sehr große Erwartungen, aber auch Befürchtungen in der Öffentlichkeit verbunden werden. Die Deutsche Forschungsgemeinschaft, also die DFG, hat zusammen mit der Nationalen Akademie der Wissenschaften und der Deutschen Akademie der Technikwissenschaften 2009 eine Stellungnahme zur Synthetischen Biologie herausgegeben, die das Forschungsfeld vorstellte. Darin wurde vorgeschlagen, dass die ZKBS ein Monitoring durchführen soll, um die wissenschaftlichen Entwicklungen auf diesem Gebiet sachverständig zu begleiten und hinsichtlich der biologischen
Sicherheit zu bewerten. Ein erster Bericht wurde bereits 2012 von der ZKBS veröffentlicht. Die damalige Bundesministerin für Ernährung, Landwirtschaft und Verbraucherschutz (BMELV), Ilse Aigner, kündigte in einem Brief an die DFG an, die ZKBS mit diesem Monitoring zu beauftragen. In einem Erfahrungsaustausch mit der ZKBS schlug sie vor, dass dies als gesetzliches Aufgabengebiet der ZKBS im Rahmen einer Novellierung des GenTG verankert werden soll. Dies ist aber bisher noch nicht erfolgt.

WW: Ich finde auch die aktuellen Diskussionen zu den „Neuen Techniken“ sehr interessant. Derzeit wird EU-weit erörtert, ob Organismen, die mit Hilfe der sogenannten „Neuen Techniken der Pflanzenzüchtung“ entwickelt wurden, vor allem durch die Oligonucleotide Directed Mutagenesis (ODM) und durch Einsatz von sequenzspezifischen DNasen wie CRISPR-Cas9, in den Anwendungsbereich der europäischen Richtlinien für gentechnisch veränderte Organismen fallen. Den Techniken ist, wenn man zum Beispiel nur das Herbeiführen von Punktmutationen betrachtet, gemein, dass die durch sie hervorgerufenen genetischen Veränderungen nicht unterscheidbar sind von solchen, die durch herkömmliche Züchtungstechniken oder natürliche Prozesse entstehen.

IK: Die Methoden und Ziele, die für biologische Forschung maßgebend sind, unterliegen ja einer ganz enormen Dynamik. Es ist sehr wichtig, dass neuartige Methoden von einem Expertengremium begleitet und bewertet werden.

WW: Ja, in diesem Zusammenhang ist auch die Diskussion zur neuen Gene-Drive genannten Methode sehr bemerkenswert. Dabei handelt es sich um eine spektakulär auch als „mutagene Kettenreaktion“ bezeichnete Methode zur Einführung von gentechnischen Konstrukten in Populationen von Organismen, die sich sexuell vermehren. Das Konstrukt bewirkt seinen eigenen Übergang vom heterozygoten in den homozygoten Zustand. Am Beispiel von Fruchtfliegen wurde gezeigt, dass sich ein solches Genkonstrukt in einem Laborstamm rasant verbreiten kann. Weltweit wird erwogen, ob Gene-Drive-Konstrukte in der Natur eingesetzt werden könnten, um zum Beispiel Insektenpopulationen zu eliminieren, die humanpathogene Erreger übertragen, wie die Tsetse-Fliege (Schlafkrankheit) oder die Gelbfieber-Mücke, die auch das Dengue- oder Zika-Virus überträgt. Gegenwärtig wurde schon erprobt, wie eine Population der Anopheles-Mücke im Labor sich so verändern lässt, dass der Erreger der 
Malaria in den Mücken nicht mehr überleben kann. Vor dem Hintergrund dieser Diskussionen und der Anfragen der Bundesländer zum Gefährdungspotenzial damit verbundener gentechnischer Arbeiten sah sich die ZKBS im Februar dieses Jahres veranlasst, die Funktion von Gene-Drive-Systemen in einer Stellungnahme grundlegend zu beschreiben und Hinweise zur Sicherheitsbewertung solcher Arbeiten zu geben.

IK: Das Arbeitsklima in der ZKBS ist selbst bei hohem Arbeitsaufkommen sehr angenehm, sowohl auf den Sitzungen als auch bei den schriftlichen Abläufen zur Vorbereitung von Beschlüssen oder bei Umlaufverfahren. Die Diskussionen sind immer sehr zielführend.

WW: Ich möchte Ihre Feststellungen aus meiner Sicht einerseits bestätigen und dann gerne auch noch ein Kompliment zurückgeben. Über die vielen Jahre mit unterschiedlicher Zusammensetzung der Experten hat sich eine herausragend offene und faire Diskussionskultur erhalten, die rein fachlich und rational stets zweckdienlich auf die Prinzipien Sicherheit und Vorsicht gerichtet ist. Dies schätze ich sehr. Ebenfalls möchte ich an dieser Stelle mit Nachdruck und Dankbarkeit festhalten, dass die ZKBS immer eine mit fachlich sehr versierten und obendrein engagierten Mitarbeiterinnen und Mitarbeitern besetzte Geschäftsstelle zur Seite hatte, ohne die die ZKBS ihre Aufgaben nicht bewältigen könnte.

IK: Vielen Dank! In der Vergangenheit haben ja häufig die zuständigen Bundesminister und Bundesministerinnen die Sitzungen der ZKBS besucht. Zum Beispiel nahm die Bundesministerin für Gesundheit, Ulla Schmidt, an der 100. Sitzung der ZKBS im Dezember 2000 teil. Im Dezember 2007 fand ein Gespräch zwischen der ZKBS und dem Bundesminister für Ernährung, Landwirtschaft und Verbraucherschutz, Horst Seehofer, und im November 2010 mit der Bundesministerin für Ernährung, Landwirtschaft und Verbraucherschutz, Ilse Aigner (Abb. 1 und 2), statt.

WW: Aber insbesondere in den 90er Jahren hat die ZKBS das Wohlwollen der politischen Leitung genossen, denn als Horst Seehofer noch Bundesminister für Gesundheit war, wurde die ZKBS sogar zu einem ausführlichen Gespräch an das BMG nach Bonn eingeladen. Und besonders in Erinnerung ist mir die 76. Sitzung im Dezember 1997 geblieben, der ein Empfang beim Bundespräsidenten Roman

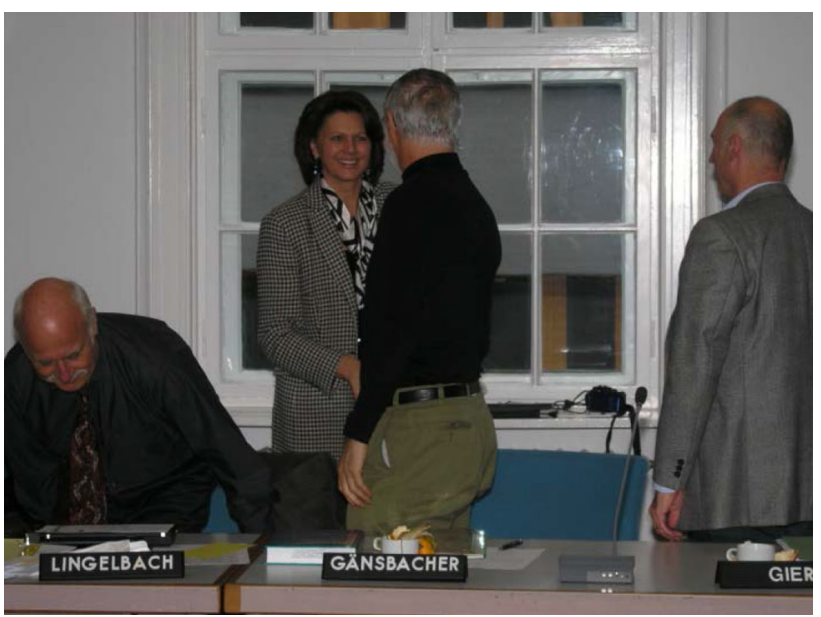

Abb. 1 Besuch der Bundesministerin für Ernährung, Landwirtschaft und Verbraucherschutz, Ilse Aigner, auf der 163. Sitzung der ZKBS am 9. November 2010. Ilse Aigner begrüßt Prof. Bernd Gänsbacher, Mitglied für den Bereich Zellbiologie (Bild: BVL)

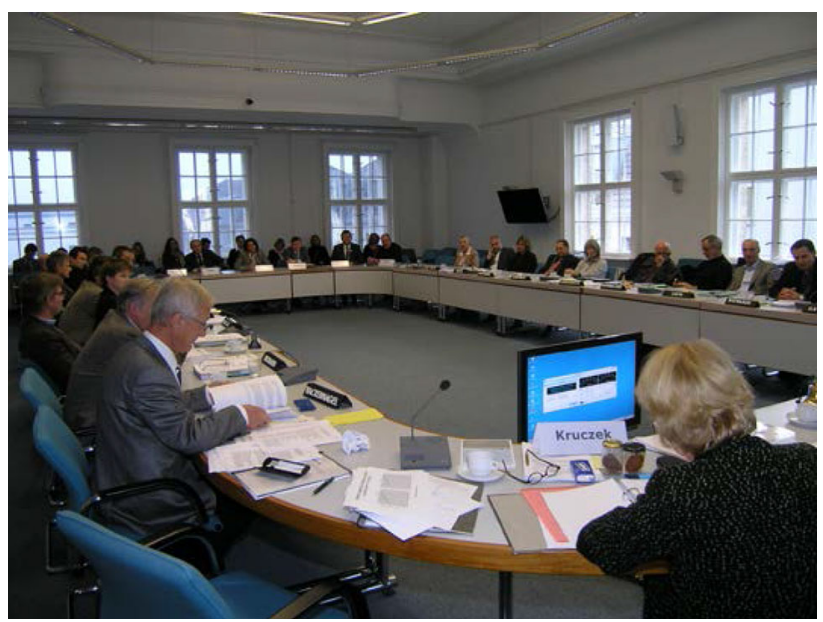

Abb. 2 Blick in den Sitzungssaal. Im Hintergrund Ilse Aigner, vorne rechts Dr. Inge Kruczek, vorne links Prof. Wilfried Wackernagel (Bild: BVL)

Herzog auf Schloss Bellevue vorausging, der sehr fachkundig mit uns diskutiert hat.

IK: Schwerpunkte der Gespräche mit den Ministerinnen oder Ministern waren immer die Zukunft der Gentechnik und die Vorstellungen zur Politikberatung durch die ZKBS. Die Bundesminister sicherten stets eine engere Zusammenarbeit zwischen dem Ministerium und der ZKBS zu.

WW: Ja, und nun hat der Bundesminister für Ernährung und Landwirtschaft, Christian Schmidt, seinen Besuch auf der 200. Sitzung angekündigt. Wir sind sehr gespannt. 


\section{Hintergrundinformationen zur ZKBS}

Die ZKBS setzt sich aus zwölf Sachverständigen, die über besondere Erfahrungen in den Bereichen der Mikrobiologie, Zellbiologie, Virologie, Genetik, Pflanzenzucht, Hygiene, Ökologie, Toxikologie und Sicherheitstechnik verfügen, und je einer sachkundigen Person aus den Bereichen der Gewerkschaften, des Arbeitsschutzes, der Wirtschaft, der Landwirtschaft, des Umweltschutzes, des Naturschutzes, des Verbraucherschutzes und der forschungsfördernden Organisationen zusammen. Dies ist im GenTG festgelegt. Für jedes Mitglied der Kommission ist aus demselben Bereich ein stellvertretendes Mitglied berufen.

Auch die Aufgaben der ZKBS sind im GenTG festgelegt. Sie prüft und bewertet sicherheitsrelevante Fragen nach den Vorschriften des GenTG, gibt hierzu Empfehlungen und berät die Bundesregierung und die Bundesländer in sicherheitsrelevanten Fragen der Gentechnik. Ihre Entscheidungen trifft sie meist auf ihren Sitzungen, in einigen Fällen auch in einem schriftlichen Verfahren. Die Mitglieder der ZKBS arbeiten ehrenamtlich und sind zur Verschwiegenheit verpflichtet. Im Wesentlichen ordnet die ZKBS Bakterien, Pilze, Parasiten und Viren auf Grundlage ihres Gefährdungspotenzials einer von vier Risikogruppen, und gentechnische Arbeiten auf Grundlage ihres Gefährdungspotenzials einer von vier Sicherheitsstufen zu. Sie empfiehlt dabei geeignete Sicherheitsmaßnahmen. Darüberhinaus gibt sie auch Stellungnahmen $\mathrm{ab}$ zu beantragten Freisetzungen oder zum beantragten Inverkehrbringen gentechnisch veränderter Organismen.

In den Anfangsjahren war das Bundesministerium für Gesundheit (BMG) für das GenTG und für die Berufungen der ZKBS-Mitglieder zuständig. Die ZKBS wird bei der Wahrnehmung ihrer Aufgaben von einer Geschäftsstelle unterstützt, die zunächst am Robert Koch-Institut (Berlin) eingerichtet war. Im Jahr 2003 ging die Zuständigkeit für das GenTG an das BMELV über. Die Geschäftsstelle wurde nun am BVL (Berlin) eingerichtet.

Die Sachverständigen werden dem zuständigen Bundesministerium, jetzt das Bundesministerium für Ernährung und Landwirtschaft (BMEL), vom Wissenschaftsrat vorgeschlagen, die Sachkundigen von ihren jeweiligen Verbänden bzw. Organisationen. Dann werden die Vorgeschlagenen vom BMEL im Einvernehmen mit den Bundesministerien für Bildung und Forschung, für Wirtschaft und Energie, für Arbeit und Soziales, für Gesundheit sowie für Umwelt, Naturschutz, Bau und Reaktorsicherheit und nach Anhörung der zuständigen Landesbehörden für die Dauer von drei Jahren in die ZKBS berufen. Eine Wiederberufung ist zulässig. 